\title{
Conserver, aménager, revitaliser les cours d'eau avec une logique naturelle
}

\author{
B. Lachat ${ }^{1}$
}

Mots-clés : Génie végétal, cours d'eau, érosion, berge, stabilisation, rivière, génie biologique, technique végétale, morphologie, aménagement.

Différents aspects de la conservation, de l'aménagement et de la revitalisation des cours d'eau sont présentés par la description des concepts, des méthodes, des matériaux et des résultats obtenus. Une place particulière est faite aux méthodes du génie végétal permettant d'atteindre des résultats hydroécologiques fonctionnels. Les fondements sont expliqués, de même que les paramètres à prendre en compte pour qu'une opération soit réussie au point de vue technique et surtout biologique.

Le génie végétal s'inscrit dans un contexte de développement durable des hydrosystèmes utilisés par l'Homme, basés sur la conservation ou la réimplantation de systèmes dynamiques rivulaires disparus. Il s'inscrit, tout comme d'autres «génies», dans la panoplie des techniques à disposition des ingénieurs pour résoudre certains problèmes d'érosion. L'ensemble de l'article donne une information et un état des connaissances actuelles sur le génie végétal. Il est cependant clairement démontré qu'il utilise les plantes vivantes comme matériaux de construction permettant d'obtenir des ouvrages extrêmement fonctionnels et durables. Il n'emploie pas les végétaux comme éléments cosmétiques de décoration d'ouvrage de génie civil !

Toute intervention sur un cours d'eau n'étant pas forcément souhaitable, l'opportunité d'une variante zéro est envisagée. La philosophie devant prévaloir aux aménagements de cours d'eau se base sur une logique naturelle qui veut que le végétal, selon les cas, soit privilégié avant le minéral : - 1) Déterminer s'il y a réellement lieu de devoir intervenir en fonction des impacts et des enjeux (variante zéro) - 2) Evaluer si les techniques végétales peuvent satisfaire à la résolution des problèmes.- 3) En cas contraire, établir si des techniques combinées peuvent pallier aux problèmes. - 4) Seulement à ce stade, appliquer une technique habituelle de génie civil pour autant qu'elle s'avère raisonnable et proportionnée.

Aménager un cours d'eau n'est pas qu'une simple affaire de calculs hydrauliques. Une connaissance des fonctions biologiques est nécessaire à l'élaboration des concepts d'intervention. Une analyse fine de l'ensemble du cours d'eau selon des critères hydroécologiques s'avère aujourd'hui indispensable. Pour mener à bien la conception d'une conservation, d'un aménagement naturel ou d'une revitalisation de cours d'eau, des directives sont proposées.

\section{Conservation, landscaping and restoration of watercourses with a natural logic}

Keywords : Soil bioengineering, erosion control, bank, protection, river, stabilization, slope, bioengineering, design.

Different aspects of preservation, management and restoration of watercourses are described with their concepts, methods, materials and results. Special emphasis is given to bioengineering which may yield very good hydroecological results.

Bioengineering is in keeping with a sustainable development of hydrosystems used by man. It is based on the conservation or, on the contrary, on the re-creation of dynamic riparian systems which are missing. Like other engineering sciences, it may solve erosion problems with great technical efficacy, but with great ecological efficiency. Living plants are used as construction materials which are arranged in specific patterns and are not employed to decorate civil engineering works.

Intervention in a river is not always necessary. A zero option is considered. The philosophy which governs interventions in watercourses must be based on a natural logic (natural development) where the vegetable is privileged before the mineral : 1) Assess the need for interventions according to impacts and values to protect (zero option) - 2) Assess if bioengineering can solve the problems. - 3) If not, assess if mixed interventions are efficient. - 4) Only at this stage should a conventional civil work technique be applied if it proves reasonable and coherent.

Regulating and landscaping a watercourse is not only a matter of hydraulic calculations. A knowledge of biological functions is needed to develop appropriate interventions. A fine analysis of the whole stream according to hydroecological criteria has become essential. For successful conservation, natural intervention and restauration of watercourses, guidelines are proposed.

1. BIOTEC Biologie appliquée SA, route de Courroux 17, CH - 2824 Vicques, Switzerland. 


\section{Introduction}

L'écosystème «cours d'eau», avec l'eau, le lit, les berges et les rives, procure quantités d'habitats diversifiés à un grand nombre d'organismes vivants. Modelés au gré des intempéries, selon la consistance des terrains qu'ils traversent, les cours d'eau présentent entre eux une multitude de différences qui confèrent à chacun sa «personnalité». Mais l'allure d'un cours d'eau dépend également des actions de l'Homme. Souvent négligés, puis trop souvent corrigés, les cours d'eau de la plupart des pays industrialisés subissent les assauts de la technologie et les tronçons demeurés encore naturels et sauvages diminuent inexorablement.

L'intérêt économique immédiat guide nos actions sur l'environnement. L'évolution de nos modes de vie et de pensée, de même que nos comportements sociaux appauvrissent, depuis une cinquantaine d'années, une multitude de milieux naturels avec pour conséquence l'uniformisation, la banalisation. Les cours d'eau n'échappent pas à cette règle.

La faune des eaux douces offre, normalement, une extraordinaire diversité, bien supérieure à celle d'autres milieux de valeur comme les prairies maigres et les haies. Cela suffit à devoir respecter et à ne plus considérer le cours d'eau comme un simple canal où s'écoule un fluide.

Malheureusement, les interventions humaines continuent de banaliser de plus en plus cette faune, qui a tendance à s'appauvrir au point de disparaître complètement de certaines régions. Les causes principales d'affectations (Fig. 1) regroupent trois grands ensembles de facteurs (d'après Lachat 1991a) :

- la qualité de l'eau (physico-chimie : pollutions chimiques, organiques, minérales, etc.) ;

- la quantité d'eau (régime hydrologique : variations artificielles, débits résiduels, etc.) ;

- la morphologie du lit et des berges (inadaptation des ouvrages hydrauliques, etc.) .

En ce qui concerne la morphologie, il reste beaucoup à faire pour marier les contraintes de l'hydraulique à celles de l'écologie : il faut développer des systèmes de protection devant permettre d'allier la sécurité à la vie.

En matière d'aménagement de cours d'eau, les idées évoluent quelque peu par rapport aux grandes interventions de correction du siècle passé poursuivies jusqu'au début des années 1990. Dans la plupart des cas, les anciens principes hydrauliques d'une absolue rigueur se transforment (mais encore trop légèrement)

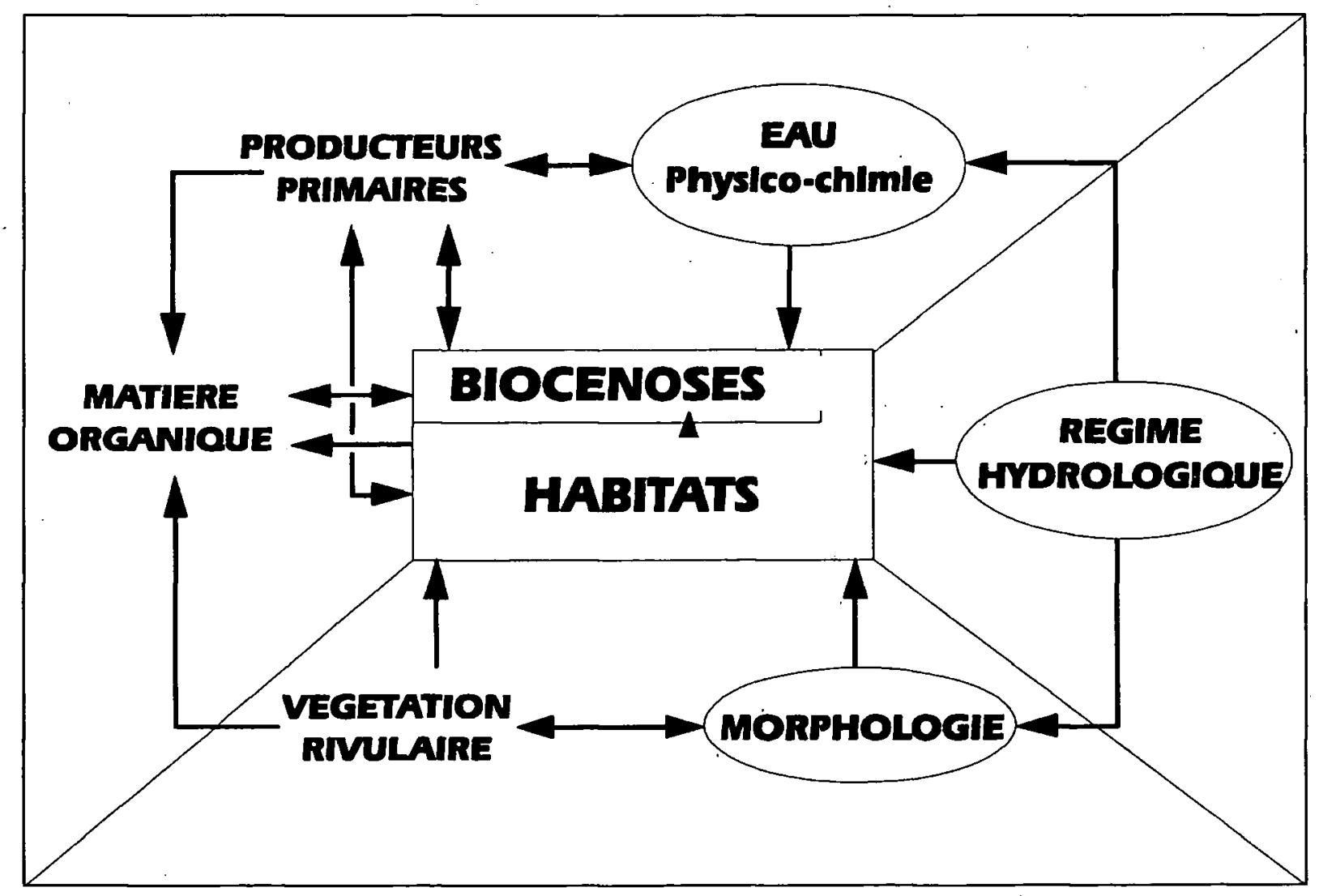

Fig. 1. Facteurs influant sur l'édifice vivant d'un cours d'eau (d'après Lachat 1991a).

Fig. 1. Factors which influence communities in a stream (according to Lachat 1991a). 
en des ensembles plus rustiques, qui n'en restent pas moins, souvent, excessivement éloignés d'un véritable cours d'eau naturel : le pavé-gazon ou les remparts de rondins remplacent les lits de plantes semi-aquatiques ; les blocs de rocher, parfois bétonnés ou cimentés, parfois percolés de terre, remplacent d'anciens murs ou des pérés lisses. Malheureusement, les enrochements recouvrent encore et toujours des sols qui, aménagés le plus naturellement possible, avec des végétaux par exemple, seraient stables à l'échelle humaine. Même si parfois elles constituent une légère amélioration par rapport à une situation antérieure (remise à ciel ouvert, décorrection), certaines de ces interventions de génie civil ou génie forestier, encore très «lourdes», sont considérées, à tort, comme des modèles écologiques parce qu'elles produisent une ou deux caches à truites ou parce que des végétaux arrivent tout de même à croître dans les interstices. Une simple analyse, ne serait-ce que végétale, fournit une preuve indubitable de la pauvreté biologique de telles interventions (Lachat 1991b). La structure du lit et les habitats sont souvent simplifiés de façon outrancière. Cela conduit à détruire les équilibres écologiques et les processus fonctionnels, dont les biomasses piscicoles peuvent être réduites dans des proportions considérables, de l'ordre de $80 \%$ (Wasson et al. 1995).

La caractéristique essentielle d'un cours d'eau naturel réside dans sa complexité et sa diversité, tant au niveau physique, chimique, que biologique. Or, l'édifice vivant que sont les biocénoses, poissons y compris, ne peut s'installer spécifiquement que si les conditions écologiques requises sont présentes.

Un cours d'eau doit donc offrir une mosaïque d'éléments physiques divers pour permettre l'établissement de communautés vivantes variées. L'hétérogénéité et la diversité sont essentielles d'un point de vue morphologique pour assurer le maintien, le développement et le respect d'un écosystème, même si cela ne simplifie en rien les calculs hydrauliques.

\section{L'édifice végétal du cours d'eau}

Mises à part les situations apicales montagnardes et alpines ou alors la traversée sur des bancs rocheux ou sur des éboulis de pentes, les cours d'eau ne possèdent pas, naturellement, de structure à base de blocs de rocher. Au contraire, selon les cas, une végétation typique se développe sur des substrats particuliers, du centre du lit mineur jusqu'au bord du lit majeur. Régie par la qualité des sols, par des conditions hydriques et hydrauliques particulières liées à la nature du cours d'eau, la végétation qui s'implante naturellement se distribue en séries plus ou moins bien marquées (Fig. 2), influencées également par des paramètres climatiques subtils, par des facteurs pédologiques variés et par des interactions biotiques.

Compte tenu d'un environnement physique assez hostile, les plantes aquatiques et rivulaires ont dû développer, du moins pour la plupart, des systèmes racinaires hautement performants, constituant ainsi des modèles de stabilisation. De plus, beaucoup de ces espèces possèdent des racines dont les tissus présentent des vides permettant la circulation des gaz, alors que la plante se trouve dans des conditions anoxiques de submersion. Mieux encore, compte tenu des difficultés qu'elles rencontrent, la plupart de ces espèces ont résolu leur problème de reproduction sexuée en développant la faculté de se multiplier végétativement (aptitude au bouturage). Voilà donc des atouts non négligeables qui vont servir les techniques du génie végétal.

Depuis plusieurs décennies, le domptage des cours d'eau (corrections, endiguements, barrage hydroélectrique, etc.) et l'état d'eutrophisation général yers lequel évolue une très grande majorité d'entre eux ont considérablement modifié l'image végétale.

En Alsace, par exemple, la végétation riveraine du Rhin dompté a beaucoup changé, alors que celle influencée par l'Ill, tout proche et encore naturel, a subi peu de transformations. En Suisse, les zones alluviales font l'objet d'études fouillées et il est inquiétant de constater que la composition floristique des associations relève de très nombreuses absences, par rapport à - des relevés effectués il y a moins de vingt ans, même si la quantité d'espèces peut rester identique. Les «vides» ont été comblés par des espèces plus courantes ou d'origine exotique. La qualité des espèces change; révélant ainsi une véritable érosion génétique. Une fois encore, on tend vers une banalisation de la végétation riveraine sous l'action de l'homme.

\section{Le génie végétal}

\subsection{Définition}

Le génie végétal se définit comme l'utilisation de plantes ou parties de celles-ci pour résoudre les problèmes de l'ingénieur dans les domaines mécaniques de la protection contre l'érosion, de la stabilisation et de la régénération des sols. La prise en compte des aspects et des fonctions biologiques est un précepte incontournable. L'aménagement naturel des cours d'eau ou la «décorrection» de ceux-ci au profit d'une revitalisation (ou restauration originelle) déborde de la notion de génie végétal et est englobé dans celle de génie biologique et de génie écologique. 
(A) L'eau libre

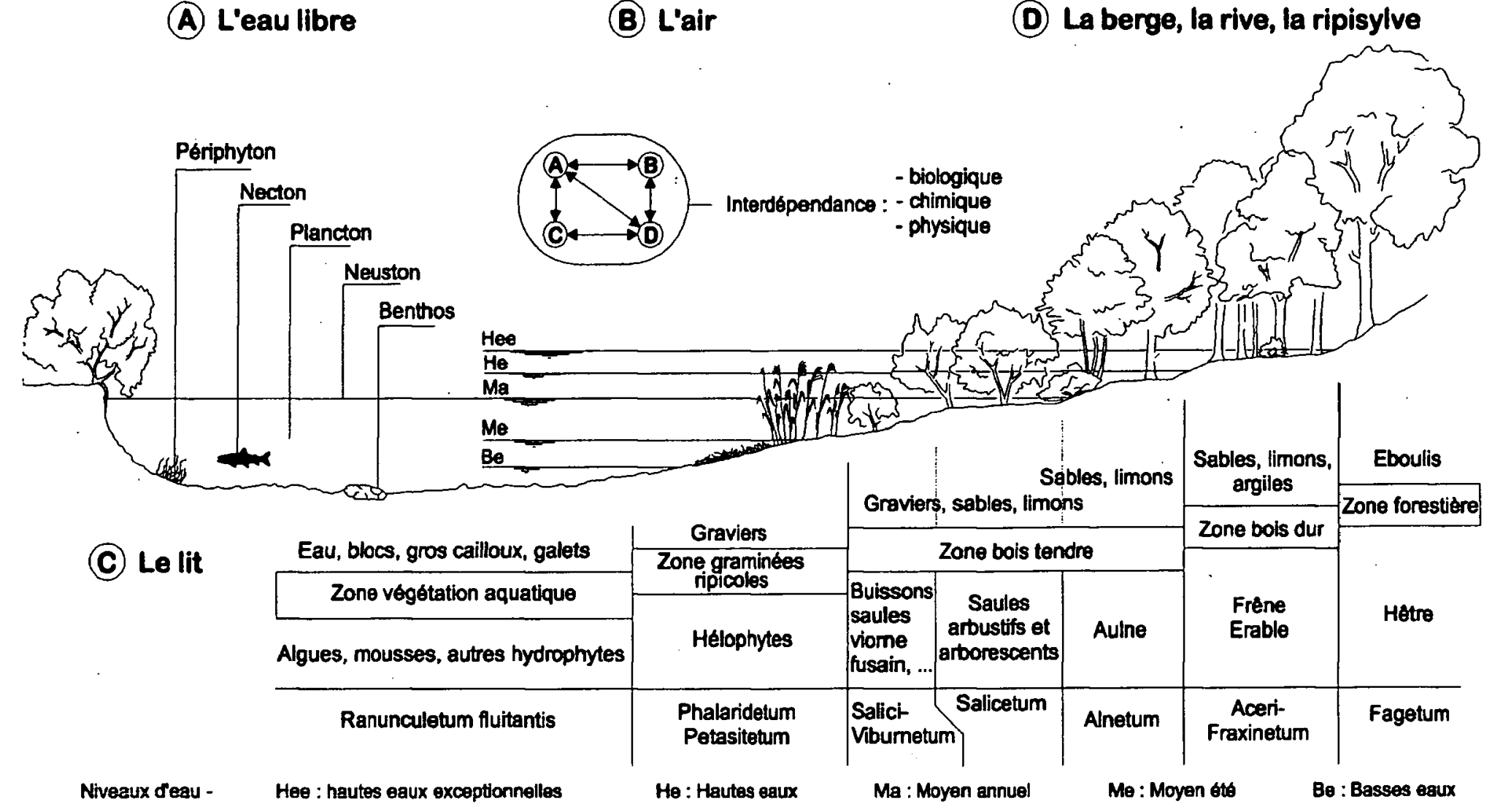

Fig. 2. Zonation transversale typique (en série) d'un bord de cours d'eau (Lachat 1991a).

Fig. 2. Typical cross section of a river with successional distribution of vegetation (from Lachat 1991a).

Les domaines d'activités possibles sont les talus (stabilisation de chemins, routes, voies ferrées, ravins, versants, glissement de terrains, etc.), les berges (cours d'eau, étang, lac, etc.) et les zones diverses à réaménager naturellement comme exprimé sur la figure 3 . Les végétaux vivants seront utilisés sous diverses formes et seront ordonnés, arrangés, composés et fixés selon des procédés particuliers. Il existe de nombreuses façons de placer et d'organiser ces végétaux ou ces parties de végétaux. Diverses méthodes ont déjà été décrites par divers auteurs (Begemann \& Schiechtl 1986, Lachat 1991a, 1991b, 1994, Lewis \& Williams 1984, Regione Emilia-Romagna 1993, Schiechtl 1973, Zeh et al. 1990).

Le génie végétal est en fait une science hybride. Sa réussite nécessite la maîtrise d'un large éventail de connaissances. Botanique, géobotanique, phytosociologie et écologie végétale en sont les principales pour ce qui est de la composante vivante. Mais physique, mécanique et dynamique des sols, connaissance des matériaux auxiliaires, géologie, pédologie et surtout hydraulique dès que l'on touche aux cours d'eau, sont autant d'outils indispensables à la réussite de l'application des techniques développées.
Ainsi, sur des modèles naturels de mécanismes de croissance connus et observés, le génie végétal développe des procédés qui permettent, parfois à grande échelle, de résoudre des problèmes de protection des sols contre l'érosion. Et c'est précisément dans le développement des techniques et leurs applications que réside son essence propre. En d'autres termes, non seulement il exploite, comme modèle, les capacités naturelles du végétal, mais il utilise ce dernier comme matériel de base à la construction d'ouvrages.

La matière végétale étant vivante, elle est par définition capable de croissance, d'évolution et sujette à des modifications aléatoires rapides, influencées par son environnement. Par conséquent, il n'est pas possible de la mettre en équation, contrairement à la matière inerte. D'autre part, des résultats positifs de mise en place de techniques végétales ne sont pas forcément reproductibles en fonction des nombreux facteurs d'influence (voir Fig. 4). Malgré tout, en matière de protection des sols riverains avec l'aide des végétaux, l'approche empirique basée sur des connaissances biologiques et hydrauliques est fiable et, vraisemblablement, une des seules valable. 


\section{Génie végétal}

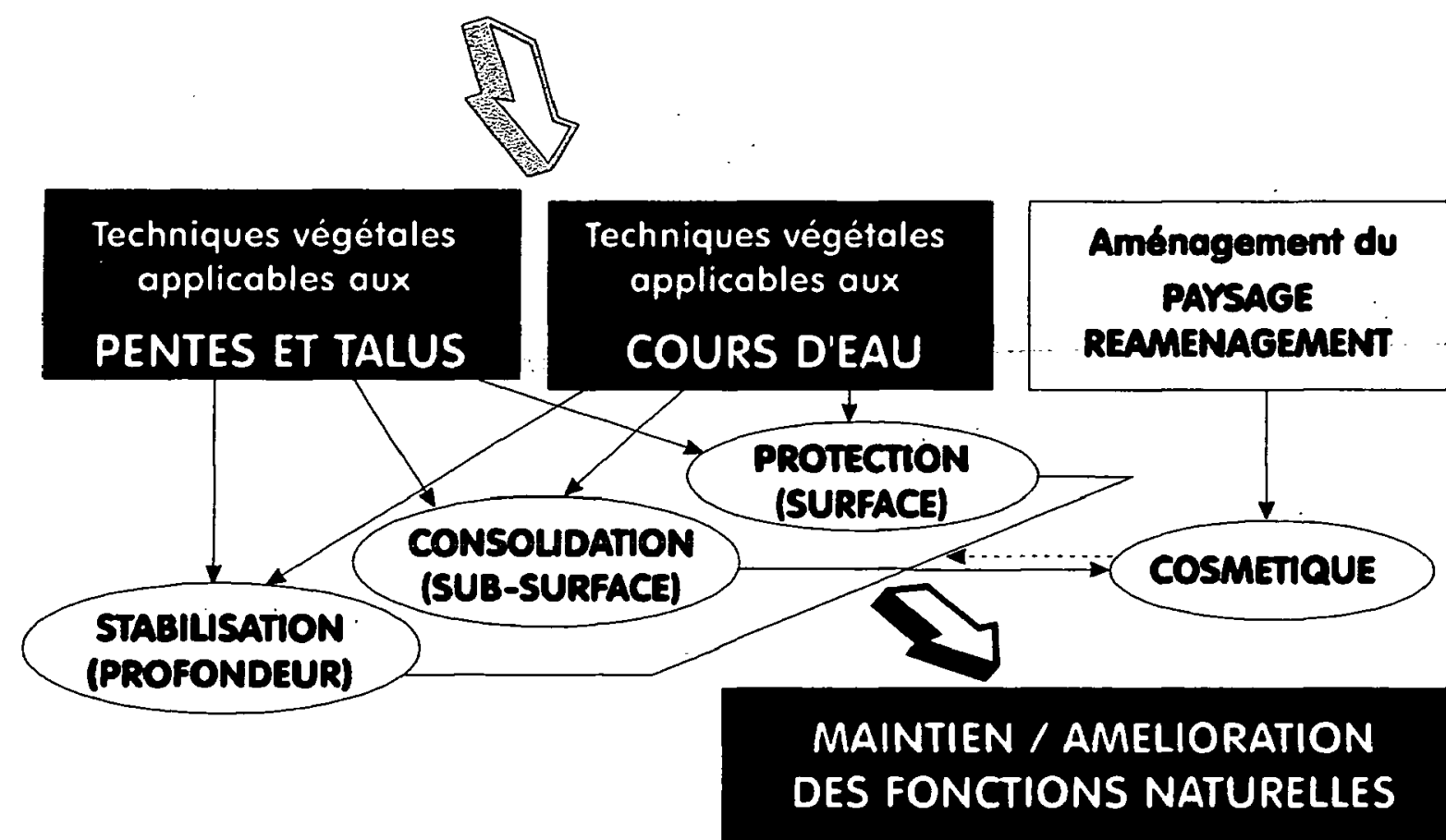

Fig. 3. Domaines et buts du génie végétal.

Fig. 3. Fields and purposes of bioengineering.

\subsection{Mode ou science?}

Le génie végétal ne date pas d'aujourd'hui, même si ce terme ne s'emploie que depuis peu (Lachat 1994).

En effet, Léonard de Vinci parlait déjà des saules et de leur utilité au bord de certains canaux. Au cours des XVII, XVIII et XIX ${ }^{\mathrm{e}}$ siècles, on a beaucoup utilisé les végétaux pour résoudre des problèmes de glissement de terrain ou d'érosion de berges de cours d'eau (Demontzey 1878 , Schlüter 1984, Thiéry 1891). Malheureusement, les connaissances sur les fonctions physiologiques des végétaux n'étaient pas tout à fait suffisantes pour comprendre certaines réponses des plantes face à leur environnement. Les moyens mécaniques manquaient aussi pour les rendre très efficaces. Vraisemblablement à cause des deux guerres mondiales et parce que la mécanisation s'intensifia alors, le génie végétal fut oublié. Il aurait pu somnoler sans doute encore longtemps si des biologistes et des ingénieurs forestiers de divers pays n'avaient pas essayé de revaloriser et d'améliorer ces techniques (Lachat 1991a, 1994, Schiechtl 1973).

Aujourd'hui, en raison de nouvelles connaissances scientifiques dans le domaine de la physiologie et de l'écologie, grâce aussi à de nouveaux matériaux comme les géotextiles et des machines modernes comme les pelles hydrauliques articulées, le génie végétal est en plein essor, à tel point que son enseignement se pratique dans de nombreux établissements de formation d'ingénieurs et d'environnemèntalistes.

\subsection{Construction d'ouvrages vivants et non pas simple végétalisation}

Si la capacité d'intégration d'un ouvrage dans le paysage est un des nombreux atouts qu'offre-le génie végétal, il ne faut pas voir dans l'utilisation de matériel vivant la seule volonté de produire un effet paysager. Les végétaux sont avant tout utilisés pour leurs rôles biologiques, bien mis en évidence par Maridet (1994), et leurs fonctions biotechniques (Gray \& Leiser 1982, Morgan \& Rickson 1995) :

- absorption des contraintes mécaniques ;

- stabilisation du sol au moyen des racines ;

- drainage du sol par évapotranspiration et formation de cavités ;

- protection contre les contraintes météorologiques (vents violents, ensoleillement excessif, glissement de neige, etc.) ;

- amélioration du sol en substances humiques. 


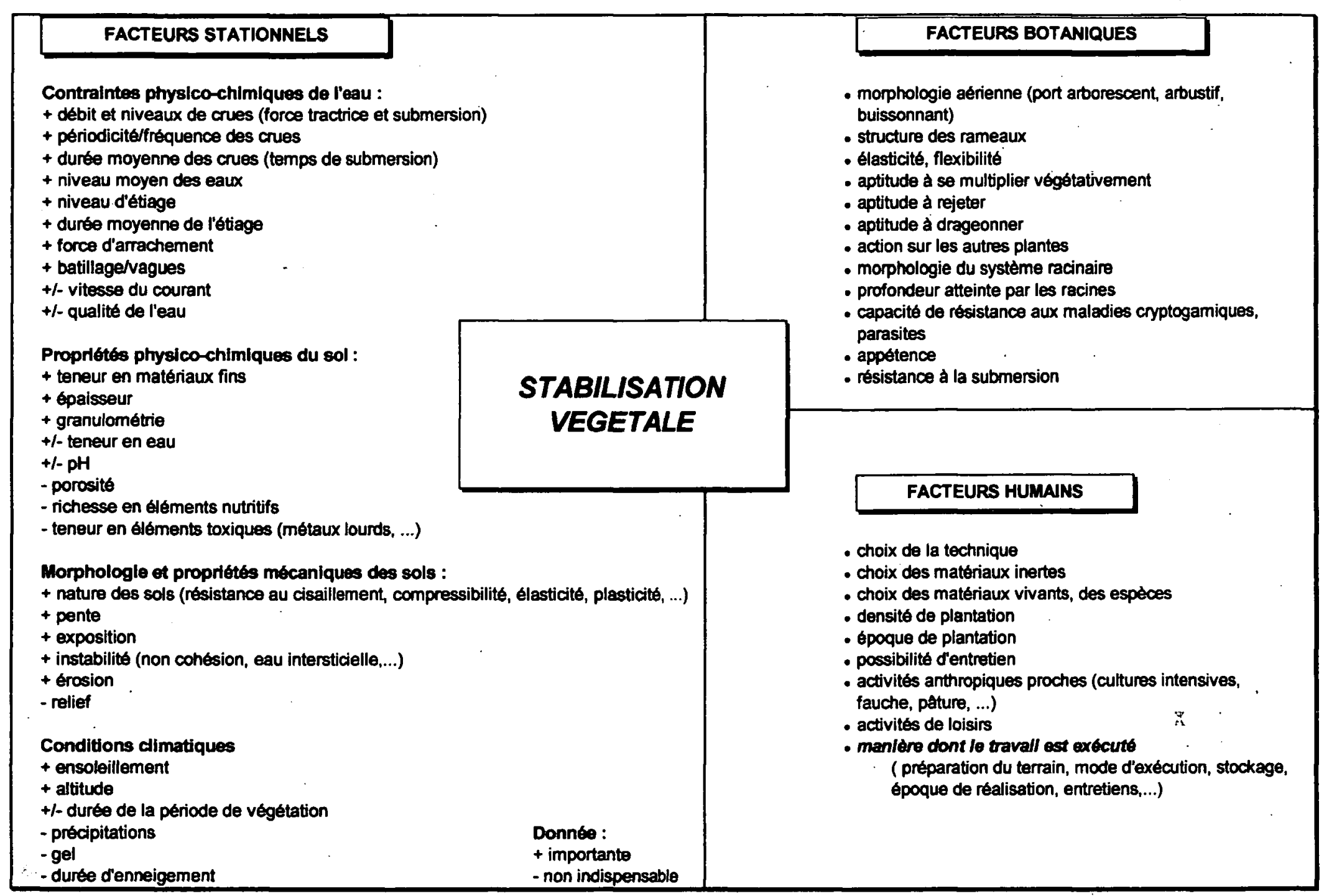

Fig. 4. Facteurs influant sur les végétaux et sur le choix d'une méthode de stạbilisation végétale (d'après Lachat 1994).

Fig. 4. Factors which influence vegetation and choice of a bioengineering technique (from Lachat 1994).

Le génie végétal est souvent assimilé à de simples plantations. Or, il n'est absolument pas comparable à cela ni à la notion qui voudrait que le végétal soit utilisé comme «cosmétique» ou comme élément décoratif d'ouvrages classiques de génie civil. Ainsi, il ne faudrait pas voir dans l'habillage de gabions ou d'enrochements, avec de grosses boutures de saule ou avec toute sorte d'autres plantes, l'application d'une technique du génie végétal digne de ce nom.

Les techniques végétales aboutissent, en réalité, à de véritables ouvrages répondant à des critères techniques et mécaniques exigeants, où des matériaux vivants (pieux, longues branches, boutures, ramilles, mottes de végétaux hélophytes, par ex.) sont utilisés comme base de la construction. Ce dernier terme est tout à fait adapté, puisqu'il s'agit réellement d'assembler et de fixer à la berge des matériaux d'origines et de qualités différentes, afin de réaliser une protection vivante, compacte, cohérente et efficace contre l'érosion, et ceci dès l'achèvement de sa réalisation.

Pour cette raison, le végétal vivant ne représente pas toujours la seule composante des ouvrages car, pour améliorer l'efficacité des réalisations, il est parfois nécessaire d'y associer des matériaux inertes ou du moins non vivants. On utilise généralement des troncs. de bois et certains types de géotextiles en fibres naturelles comme le jute, le coco, etc. (Lachat 1984, 1986, 1994, Schiechtl 1973). Leur rôle est en particulier d'accroître la résistance de l'ouvrage lors de la phase initiale de son existence, pendant laquelle les racines des végétaux n'ont pas encore acquis un développement assez performant.

A long terme, ces matériaux auxiliaires sont, la plupart du temps, destinés à se décomposer et sont peu à peu remplacés par les organes souterrains des végétaux en croissance. 
Si des matériaux inertes tels que des blocs de roche sont utilisés, c'est généralement pour avoir une influence durable sur le courant par la création d'ouvrages transversaux à l'exemple de seuils ou d'épis. Leur pérennité est évidemment souhaitée. Mais dans un projet émanant du génie végétal, l'utilisation de la matière minérale se limite généralement à la réalisation d'ouvrages complémentaires aux techniques végétales et constitue rarement une composante à part entière de l'ouvrage principal de stabilisation.

Il existe cependant des exceptions à cela, notamment dans le cas d'une trop forte artificialisation du cours d'eau ou lorsque les berges subissent des submersions constantes d'une forte amplitude liées à des marnages ou aux marées par exemple. Le marnage régulier, la vase et le sel peuvent constituer des facteurs limitants à l'utilisation des végétaux seuls. On développera, dans ce cas, des techniques mixtes (ou combinées).

\subsection{Adaptation des techniques de base à chaque projet}

Toute implantation de végétation, y compris celles de végétaux fonctionnant comme éléments de construction, doit être précédée d'une analyse des conditions de croissance qu'offrira la station (Figs 4 et 5).

Cette appréhension des paramètres se fait, en général, complètement sur le terrain et consiste d'une part en une approche globale (Fig. 4) et d'autre part dans la saisie de données essentielles pour l'élaboration du projet. D'autres résultats importants pour la nature des ouvrages s'obtiennent en laboratoire ou par calcul.

L'expérience de terrain et surtout la connaissance des phénomènes qui s'y passent ainsi que des êtres vivants qui l'habitent sont absolument fondamentales. Il est inutile de vouloir implanter des saules si l'on ne connaît ni les espèces, ni leurs exigences édaphiques, ni leur étage de croissance en relation avec les niveaux d'eau, etc.

D'une manière générale, l'élaboration d'une carte synthétique aide beaúcoup à la compréhension de l'état existant et à diriger l'aménagement futur. Toutefois, elle n'est pas indispensable mais l'appréhension de la composition botanique sur le site à travailler et sur ses abords est incontournable pour détecter la présence des espèces capables de jouer un rôle actif en protection des berges.

De plus, la représentation des objets conduisant à des phénomènes modifiant l'écoulement et provoquant des érosions peut être un outil précieux pour comprendre et élaborer des modes d'implantation de la végétation (Fig. 5).
Le choix des végétaux est déterminant dans la réussite des constructions. La règle est de travailler avec des essences indigènes, adaptées aux conditions locales de croissance. Pour des interventions sur des cours d'eau dont les rives sont encore habillées d'une végétation naturelle, la meilleure solution consiste à prélever les matériaux sur place en veillant à ne pas prendre des espèces exotiques. De cette manière, on a la meilleure garantie possible que les essences utilisées soient fonctionnelles pour les ouvrages.

Les cultivars de peupliers noirs (Populus $x$ nigra), même s'ils peuvent se bouturer facilement, sont à déconseiller en raison de leur enracinement superficiel et de leur port élevé qui les rendent trop souvent sujets au déchaussement et qui fragilisent la berge. Le volume des racines par rapport aux tiges est assez explicite de la qualité de fixation des sols par les végétaux (Tableau 1). Les arbres qui ont un rapport inférieur à «1» ne de-

Tableau 1. Rapport $(R)$ volume racines / volume tiges chez quelques espèces ligneuses pionnières spontanées (d'après Schiechtl 1973).

Table 1. Ratio (R) between roots volume and stems volume for some woody pioneer and spontaneous species (according to Schiechtl 1973).

\begin{tabular}{llc}
\hline Nom Latin & Nom vernaculaire & $\mathrm{R}$ \\
\hline Salix glabra & Saule glabre & 2.4 \\
Viburnum lantana & Viorne lantane & 2.3 \\
Salix elaeagnos & Saule drapé & 1.8 \\
Salix nigricans & Saule noircissant & 1.8 \\
Salix appendiculata & Saule à grandes feuilles & 1.7 \\
Alnus viridis & Aulne vert & 1.6 \\
Fraxinus excelsior & Frêne élevé & 1.5 \\
Salix purpurea & Saule pourpre & 1.5 \\
Lonicera xylosteum & Chèvrefeuille des haies & 1.3 \\
Ligustrum vulgare & Troène vulgaire & 1.2 \\
Rhamnus cathartica & Nerprun purgatif & 1.2 \\
Acer pseudoplatanus & Erable sycomore & 1.1 \\
Populus tremula & Peuplier tremble & 1.1 \\
Rubus idaeus & Framboisier & 1.1 \\
Hippophae rhamnoides & Argousier faux nerprun & 1.0 \\
Rosa canina & Eglantier & 0.9 \\
Cornus sanguinea & Cornouiller sanguin & 0.7 \\
Berberis vulgaris & Epine-vinette & 0.6 \\
Salix alba & Saule blanc & 0.5 \\
Salix triandra & Saule à trois étamines & 0.4 \\
Populus nigra & Peuplier noir & 0.4 \\
\hline
\end{tabular}


234

B. LACHAT

(8)

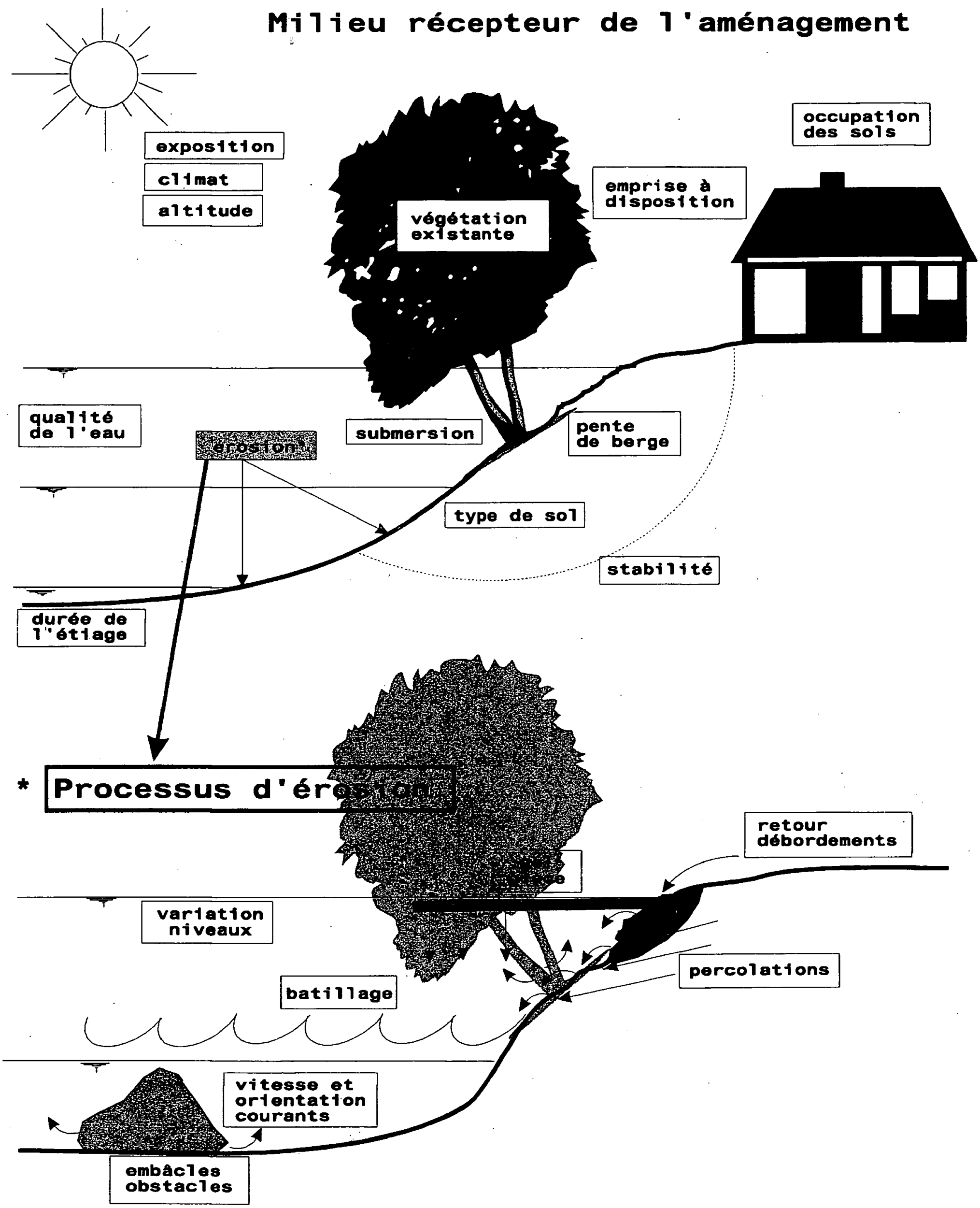

Fig. 5. Quelques paramètres influant sur le milieu récepteur does ouvrages et processus d'érosion-possibles.

Fig. 5. Some parameters having an influence on the site which must receive the work and illustration of possible erosion causes. 
vraient pas être placés sur la berge lors d'aménagement. Il en est de même des buissons ou arbustes ayant un rapport inférieur à «0,5». Ceci dépend bien sûr de l'énergie du cours d'eau, des conditions locales environnantes et des buts poursuivis.

Si une catégorie de végétaux ligneux, les saules par exemple, viennent à manquer, notamment par l'absence ou la modification de la ripisylve, et afin de garantir un approvisionnement de qualité avec les espèces spécifiées dans les projets, il est parfois nécessaire de constituer des plantations pépinières. Comme la plupart des saules se bouturent très facilement, la production de baguettes pour les ouvrages s'avère rapide et fonctionnelle si l'on prend soin d'utiliser des sols adaptés.

Dans un aménagement, il est toujours recommandé, de varier les essences, que ce soit les espèces de saule, s'il s'agit de matériaux devant être aptes à rejeter, ou les genres si des plants à racines nues peuvent entrer en ligne de compte. Cette recommandation n'a pas pour seul but de satisfaire à des critères de diversité biologique, mais elle augmente également les chances d'une bonne reprise de la végétation, et de ce fait, favorise un bon comportement de l'ouvrage. Respecter les facteurs stationnels signifie également que les essences seront distribuées sur la berge en respectant le principe des séries végétales, réparties en fonction des contraintes hydriques et hydrauliques. Ceci exige évidemment des connaissances indispensables en botanique.

Le choix des végétaux doit également tenir compte de leur aptitude à être utilisés comme éléments de construction, qu'il s'agisse de leur aptitude à rejeter, à drageonner, de leur flexibilité, de leur mode de croissance ou de leur développement futur.

L'amélioration considérable de l'efficacité des techniques face aux pratiques du siècle passé ou du début $\mathrm{du} \mathrm{XX}^{\mathrm{e}}$ siècle permet de proposer leur réalisation sur des linéaires de berge importants. On peut étendre leur application à de nombreux types de cours d'eau différents, que ce soit en relation avec le régime hydraulique, le faciès, la nature des berges et des substrats ou encore l'origine des dérangements constatés. De plus, la remise à ciel ouvert d'un cours d'eau ou la décorrection d'un autre peut (ou devrait) se pratiquer avec des techniques végétales adaptées.

De nouvelles méthodes ont également été développées, de telle sorte qu'il est maintenant possible de trouver une solution idéale pour de nombreux cas particuliers (voir exemples de réalisations figures $6 a-6 d$, $7 \mathrm{a}-7 \mathrm{~d}, 8 \mathrm{a}-8 \mathrm{e})$.
Les techniques de base des constructions végétales peuvent se regrouper en diverses catégories selon les auteurs (voir Lachat 1994, Schiechtl 1973). Les plus connues sont :

- le bouturage;

- le tressage;

- le fascinage (simple ou à double rangée de pieux, constitué de branches de saules ou également de végétaux hélophytes);

- les couches de branches à rejets ou garnissage;

- les lits de plants et plançons;

- le peigne;

- le caisson en rondins, végétalisé, à paroi simple ou double;

- le treillage en rondins (plutôt utilisé pour des glissements de terrain).

Il est bien sûr exclu de concevoir et réaliser un bon projet en calquant simplement un même aménagement type sur tous les cas d'érosion qui se présenteraient.

Dans tout projet, il y a lieu aussi de déterminer quelles berges ou rives doivent être conservées dans leur état et lesquelles doivent être améliorées totalement ou partiellement. Suivant le contexte, les contraintes environnantes et les valeurs réelles à protéger; des berges qui s'érodent ne doivent pas obligatoirement être modifiées.

\section{Avantages, capacités et limites}

\subsection{Avantages}

Les avantages procurés par les techniques végétales peuvent se résumer, de façon non exhaustive, comme suit :

- efficacité de stabilisation dynamiquement croissante ;

- souplesse des ouvrages et résistance (ancrage dans la berge et non appui contre elle ; tensions d'arrachement ou de glissement reprises en traction) ;

- actions hydromécaniques de la végétation dans le sol : interception, absorption et transpiration d'eau, augmentation de la cohésion, effet de cintrage, ralentissement des vitesses d'écoulement ;

- relations cours d'eau / nappes phréatiques non perturbées ;

- impacts de chantiers minimaux ;

- amélioration de l'autoépuration du cours d'eau au niveau des racines ;

- participation et augmentation de la biodiversité et de la valeur du milieu (support, abris, nourriture) ; 

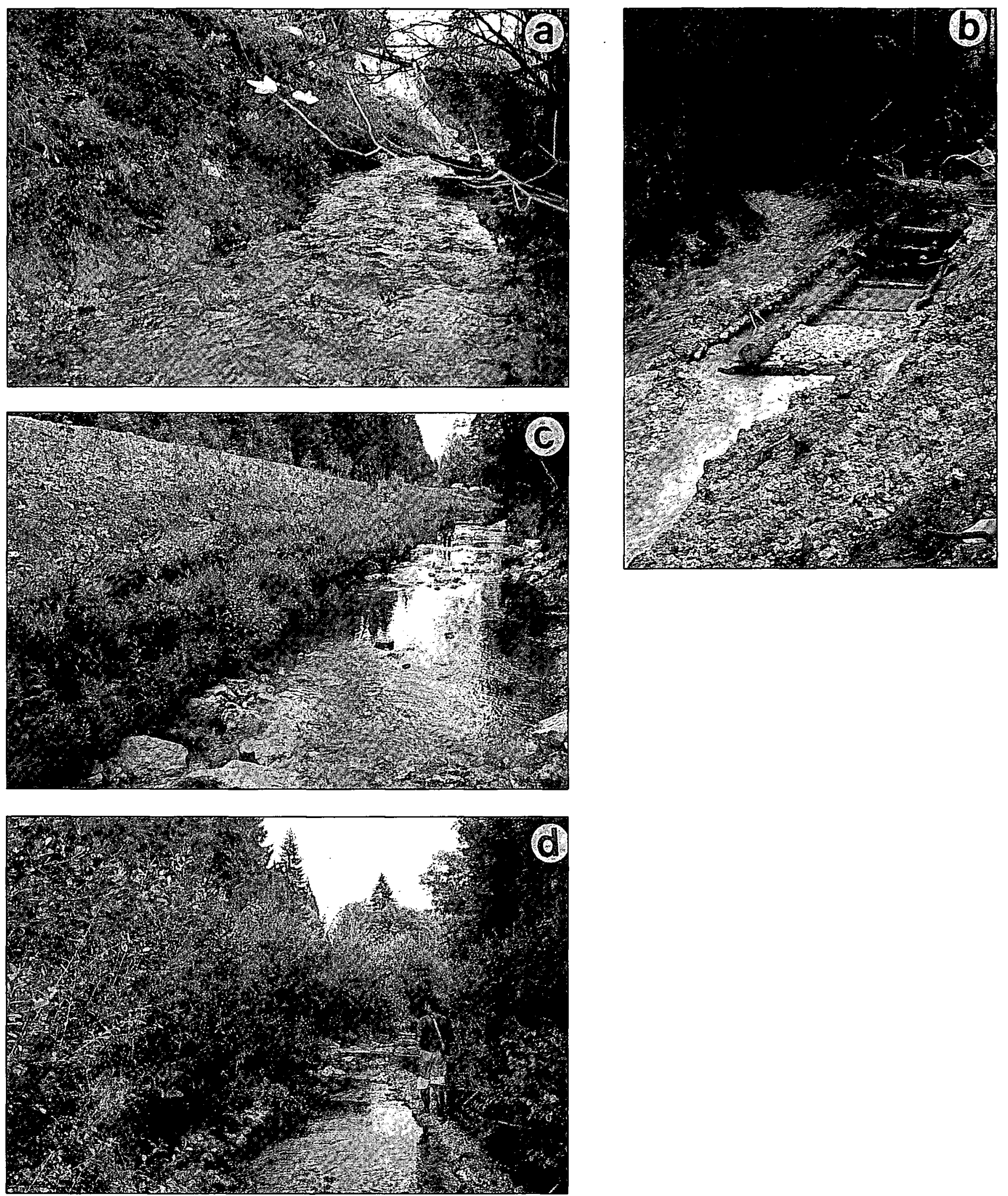

Fig. 6. La Trame à Tavannes (Jura suisse).

a. Etat de la berge avant les travaux. L'érosion et des glissements de terrain menaçaient une route en sommet de berge. - 29.10.92. (Photo : B. Lachat). b. Mise en place de l'armature du caisson qui abritera les matériaux terreux, les boutures et les plants forestiers. - 26.11.92. (Photo : P.A. Frossard). c. L'ouvrage fini présente un aspect compact et solide. La végétation se développe et fera le reste. - 17.6.93. (Photo : B. Lachat). d. Evolution de l'ouvrage. Ce type de technique s'emploie aussi sur de grands cours d'eau. - 19.8.94. (Photo : B. Lachat)

Fig. 6. The Trame river at Tavannes (Switzerland - Jura).

a. State of the riverbank before working. A main road was in danger of collapsing due to erosion and slope failure. -29.10 .92 . b. Setting earth- and rock-filled double log crib planting with saplings and cuttings. - 26.11.92. c. The finished structure offers a compact and strong aspect. The vegetation will do the rest, during its growth. - 17.6.93. d. Development of the bioengineering technique. It can be used on large watercourses too. - 19.8.94. 

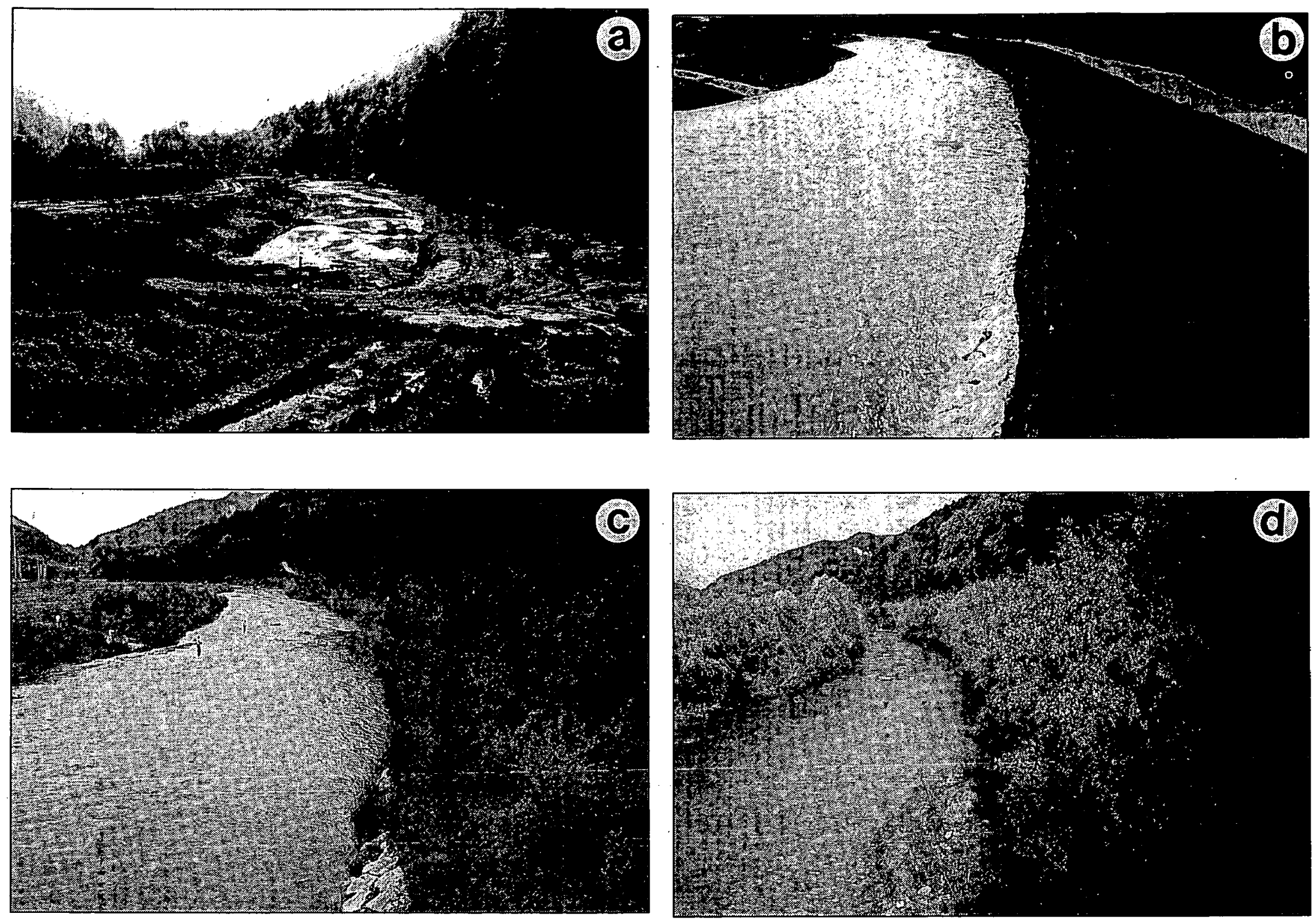

Fig. 7. La Birse à Soyhières (Jura suisse).

a. Creusage du nouveau lit. - 1.6.84. (Photo : B. Lachat). b. Technique végétale de protection de la berge, utilisant 3 types de géotextiles différents pour pallier aux problèmes contraignants de saisons et de délais de réalisation des travaux. La protection a été conçue et basée sur l'utilisation de graminées à action immédiate. La rivière coule ici pour la première fois dans son nouveau lit avec des berges protégées par des végétaux herbacés. - 27:11.84. (Photo : B. Lachat). c. Les espèces ligneuses ont participé ultérieurement à la stabilisation générale. - 19.6.88. (Photo : B. Lachat). d. Avec une variabilité du tracé, des berges et des structures végétales, la rivière offre un aspect naturel et des fonctions biologiques réelles. Un des objectifs du génie végétal est d'arriver à ce qu'on ne voit pas qu'il y a eu une intervention ! - 31.5.92. (Photo : B. Lachat).

Fig. 7. The Birse river at Soyhières (Swiss Jura).

a. Construction of the new channel. - 1.6.84. b. The river bank was protected with bioengineering methods using 3 woven geotextiles. This river bank was stabilized with a cover of herbaceous vegetation. Trees and shrubs were planted in the Spring of 1985 . Here the river can be seen flowing for the first time in its new bed. - 27.11.84. c. These trees and shrubs subsequently helped stabilize the river bank as they grew. The landscape design and new vegetation gave a natural aspect to the river. The Birse soon recovered its original biological functions. - 19.6.88. $\mathrm{d}$. Eight years after the construction, it will show that no intervention has been made. This is one purpose of bioengineering. Overflows have brought willow twigs and other vegetation which have enriched the riparian forest. ! - 31.5.92. 

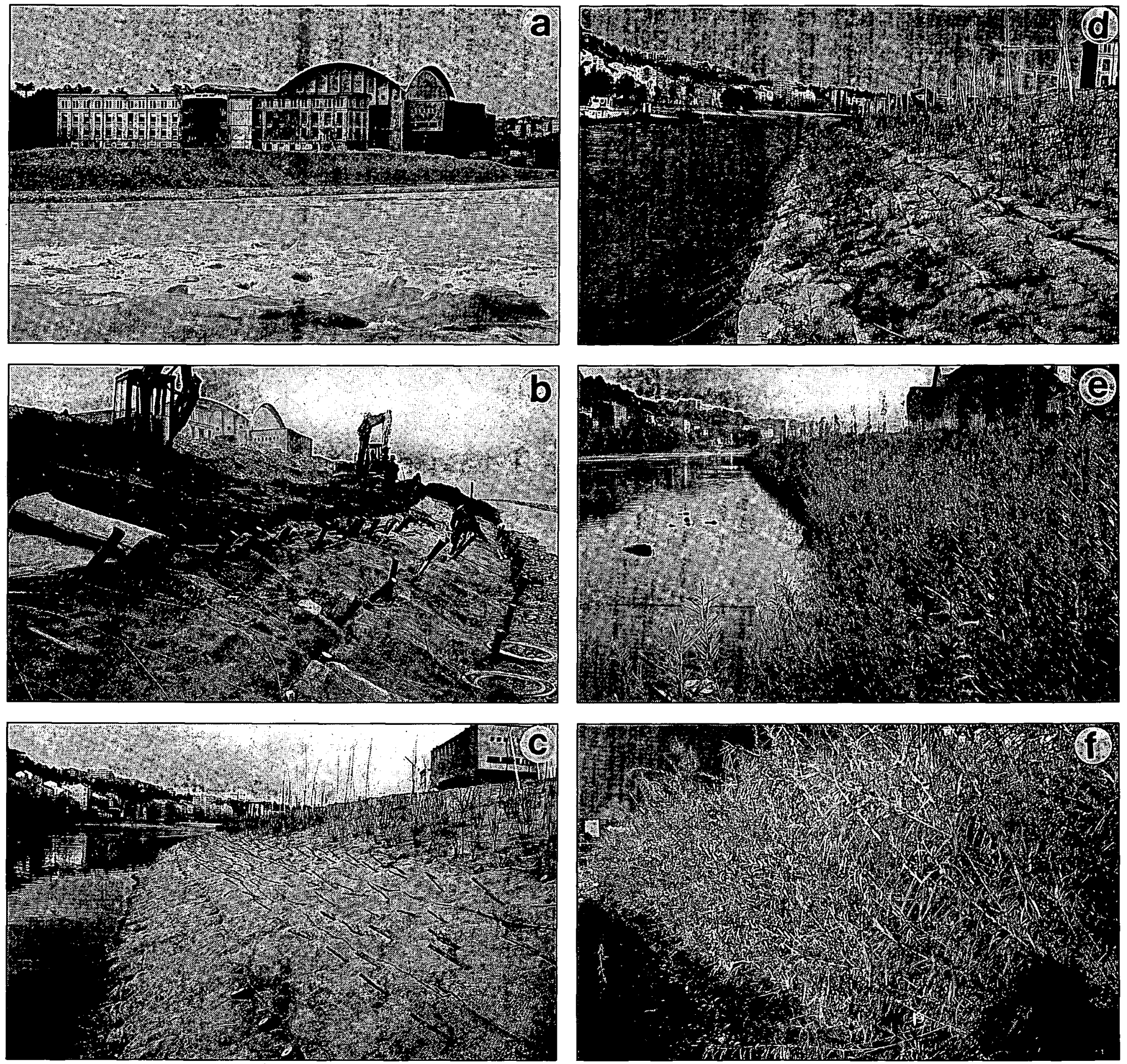

Fig. 8. Le Rhône à Lyon (France).

a. Etat du remblais à protéger dans le Rhône avant les travaux de techniques végétales. - 16.9.93. (Photo : B. Lachat). b. Mise en place des couches de branches de 5 espèces de saules, recouvrement avec un géotextile putrescible et fixation à l'aide de pieux de saules. - 24.1.94. (Photo : B. Lachat). c. Etat de l'ouvrage à la fin des travaux où l'on distingue bien les séries de végétation mises en place. -14:3.94. (Photo: B. Lachat). d. Un mois après la fin des travaux, les végétaux montrent toute leur potentialité de croissance. La technique utilisée a aussi été conçue pour résister au batillage lors du passage des bateaux. - 11.4.94. (Photo : B. Lachat). e. Les techniques végétales déploient toute leur efficacité. - 4.7.94. (Photo : B. Lachat). f. Quelques mois après, la végétation s'est développée formant des massifs buissonnants inattaquables par l'eau. Le saule des vanniers (Salix viminalis) domine sur les autres. Même sur de gros cours d'eau, les techniques végétales sont utilisables. 8.9.94. (Photo : B. Lachat).

Fig. 8. The Rhône river at Lyon (France).

a. State of the material deposition to be stabilized, before working with soil bioengineering methods. -16.9 .93 . b. Setting brush mattress with five kinds of willows. Recovering with a coconut fiber mesh and fixation with willow live poles. - 24.1.94. c. The finished structure offers a typical «natural» cross section with successional distribution of vegetation. -14.3.94. d. One month after the end of the work, vegetation show its capabilities of growth and its potentialities of stabiliżation. The bioengineering used has been elaborated to resist to the wake of boats. 11.4.94. e. Bioengineering spread out all their efficiency. - 4.7.94. f. Some months later, vegetation forms clumps of shrubs which are erosionresistant. Common Osier (Salix viminalis) is dominated. Even on big watercourses bioengineering may be employed. - 8.9.94. 
- réservoir génétique et patrimonial;

- augmentation de la teneur en oxygène de l'eau et de la fraîcheur des eaux (ombre, rejet $\mathrm{O}_{2}$ );

- diminution de la turbidité de l'eau et du colmatage; - diminution des plantes envahissantes (algues,...);

- conservation et embellissement du paysage, inscription parfaite dans le site ;

- intérêt pédagogique ;

- sur travaux normaux : gains financiers sur les techniques habituelles généralement de 40 à $90 \%$;

- pour travaux difficiles (artificialisation élevée et/ou forte ampleur de l'érosion) : coûts identiques ou légèrement plus élevés que les techniques classiques mais les résultats «écologiques» sont nettement meilleurs et les ouvrages sont vivants donc pérennes ;

- fournitures souvent sur place, transports simplifiés (gains énergétiques) et piste de chantier inutile ;

- solutions limitant les procédures et les recours (avantages financiers importants) ;

- exploitation des produits possibles (permet un revenu).

\subsection{Limites du génie végétal}

Malgré les nombreux avantages que procurent les techniques végétales, on rencontre quelques désavantages et parfois des facteurs limitants (Lachat 1994, Zeh 1990), directement liés aux conditions de croissance des végétaux :

- selon le type de technique, l'efficacité de la stabilisation peut ne pas être maximale dès la finition de l'ouvrage. Cependant, les limites peuvent être repoussées par l'utilisation judicieuse de géotextiles et par une mise en œuvre plus conséquente des moyens de fixation ;

- il existe des facteurs limitants dont les principaux sont : altitude, fortes pollutions régulières, manque de lumière, dureté des sols, substrat rocheux, sel, marée, batillage important, pente et énergie élevées. Dans certains cas extrêmes d'érosion, ces techniques ne sont pas applicables sans nécessiter des moyens financiers et techniques équivalents aux constructions habituelles du génie civil;

- quelques types de réalisation sont exigeants en main-d'œuvre ;

- il est nécessaire d'avoir une main-d'œuvre formée ainsi qu'un encadrement scientifique et technique compétent et sérieux. Les entreprises spécialisées et la main-d'œuvre qualifiée sont encore peu nombreuses à ce jour ;

- selon la petitesse du cours d'eau, et le développement végétal obtenu, il est parfois nécessaire d'entretenir régulièrement, toutefois de façon très étagée dans le temps ;
- même si l'efficacité est présente, les résultats ne sont pas toujours visibles immédiatement et obligent à patienter une période de végétation.

Les torrents de montagne, par exemple, regroupent typiquement plusieurs facteurs limitants qui rendent parfois l'application des techniques végétales très problématique : substrat rocheux, limite altitudinale de la végétation, régime torrentiel, pente du cours d'eau et énergie élevées, phénomène puissant de laves torrentielles.

Les facteurs humains précisés sur la figure 4 sont tout aussi déterminants dans la réussite des ouvrages de protection de berges. Les domaines de connaissances de base citées au $\S 3.1$ sont indispensables, à des degrés divers, à tous les acteurs, du concepteur au réalisateur. Mais les connaissances seules ne suffisent pas. Il faut aussi développer des compétences dans ces divers domaines et dans les applications pratiques.

La nature fort complexe des cours d'eau ne permet pas et n'autorise pas les simplifications.

\section{Remarques finales}

Le génie végétal s'inscrit, tout comme d'autres «génies», dans la panoplie des techniques à disposition des ingénieurs pour résoudre certains problèmes d'érosion. Le fait d'utiliser des plantes vivantes conduit à obtenir des ouvrages extrêmement fonctionnels. et durables. Les végétaux ne sont pas des éléments cosmétiques mais bien des matériaux de construction.

Aménager un cours d'eau n'est pas qu'une simple affaire de calculs hydrauliques. Une connaissance des fonctions biologiques est nécessaire à l'élaboration des concepts d'intervention. Une analyse fine de l'ensemble du cours d'eau selon des critères hydroécologiques s'avère aujourd'hui indispensable. Pour mener à bien la conception d'un aménagement naturel ou d'une revitalisation, on peut émettre les directives suivantes :

- Le cours d'eau, dans son ensemble, c'est-à-dire avec son lit, ses berges, ses rives, ses nappes et son bassin versant forme un tout et doit être analysé globalement.

- Le cours d'eau est un élément naturel dynamique d'un point de vue chimique, physique et biologique ; il faut le garder libre d'immissions polluantes et d'obstacles artificiels perturbants infranchissables.

- Le cours d'eau a besoin de place; on s'efforcera de lui donner l'espace nécessaire au maintien de son comportement sauvage.

- Le cours d'eau naturel tend à montrer une diversité maximale d'habitats et de faciès fonctionnels. On s'at- 
tachera à maintenir, reconstituer ou augmenter cette diversité.

- Chaque cours d'eau possède sa «personnalité», ses spécificités ; on adaptera la solution d'aménagement à cette individualité.

- Sur un cours d'eau, les travaux d'entretien et d'aménagement se feront de façon pondérée en accord avec les cycles faunistiques et floristiques.

- Si des mesures techniques doivent être prises pour lutter contre des dégâts dus aux cours d'eau, on préférera des solutions hydroécologiques et biotechnologiques aux autres. La philosophie devant prévaloir à ce principe s'appuie sur une démarche logique et s'établit sur les bases du tableau 2 .

Si l'aspiration de départ était de résoudre techniquement un problème d'érosion; le rôle «d'ingénieur» est sans doute rempli. Mais celui du «biologiste» peut ne pas être atteint. En effet, comme divers auteurs l'ont déjà spécifié (Leuzinger \& Lachat 1995), la réussite complète d'un ouvrage de génie biologique ne peut pas uniquement s'envisager sous les aspects techniques. La satisfaction ne sera totale que si le milieu récepteur de l'ouvrage garde ou acquiert, en plus, des potentialités (et donc des valeurs) écologiques supérieures à son état initial et supérieures à ce qu'un ouvrage classique aurait pu apporter.

Le génie biologique doit résoudre des problèmes techniques avec élégance ; il doit surtout être une composante vivante et valorisante du milieu naturel. Le génie végétal construit avec des végétaux vivants organisés selon des techniques particulières. Il n'est pas comparable à de simples plantations et n'est surtout pas une activité cosmétique pour des ouvrages de génie civil. Il est une imitation de la Nature, accélérée artificiellement pour lutter contre des problèmes graves d'érosion.

Les avantages très nombreux, notamment financiers, esthétiques, écologiques, etc., doivent maintenant conduire à systématiser les méthodes du génie végétal lorsqu'il y a nécessité de lutter contre les érosions des berges. Les résultats probants obtenus, en Europe notamment, permettent d'affirmer que ces techniques sont généralisables sur différents types de cours d'eau et dans différentes régions.

La connaissance des végétaux et de la mécanique des fluides, entre autres, sont indispensables à la réussite des ouvrages. Ceci n'est cependant pas suffisant : il est nécessaire d'acquérir également des compétences dans ces domaines au niveau de leur réalisation.

L'engouement suscité par des résultats spectaculaires va certainement provoquer des échecs chez ceux qui ne maîtrisent pas totalement les disciplines nécessaires. Nous osons croire que cela ne discréditera pas trop les techniques végétales, car, on aurait pu, depuis longtemps, abandonner d'autres techniques plus minérales dont les échecs sont très nombreux, parfois cuisants et très coûteux.

Face à la Nature et aux éléments qui la composent, il faut savoir rester modeste et humble. De toute évidence, le passé nous a montré (et le présent nous le montre tous les jours) qu' aucune technique de protection, même très chère, n'est éternelle. Alors pourquoi pas le génie végétal, qui s'apparente plus à la logique naturelle que toute autre technique?

Tableau 2. Philosophie à adopter face aux problèmes d'érosion en cours d'eau.

Table 2. Philosophy to carry facing erosion problems on watercourses.

1) Déterminer s'il y a réellement lieu de devoir intervenir, en fonction des impacts et des enjeux (variante zéro)

2) S'il faut intervenir évaluer si les techniques végétales peuvent satisfaire à la résolution des problèmes.

3) En cas contraire, établir si des techniques combinées peuvent pallier aux problèmes.

4) Seulement en dernier stade, appliquer une technique habituelle de génie civil pour autant qu'elle s'avère raisonnable et proportionnée. 


\section{Travaux cités}

Begemann W. \& Schiechtl H. M. 1986. - Ingenieurbiologie. Handbuch zum naturnahen Wasser- und Erdbau. Bauverlag, Wiesbaden und Berlin : $261 \mathrm{p}$.

Demontzey P. 1878. - Etude sur les travaux de reboisement et de gazonnement des montagnes. Paris. Imprimerie nationale.

Florineth F. 1982. - Erfahrungen mit ingenieurbiologische Massnahmen bei Fliessgewässer im Gebirge. In Ökologie von Fliess. gewässern-Ingenieurbiologische Sicherungsmassnahmen. Techn. Univ. Wien. Landschaftswasserbau. 3, : 243-262.

Gray D.H. \& Leiser A.T. 1982. - Biotechnical slope protection and erosion control. Krieger (Ed.) : $271 \mathrm{p}$.

Lachat B. 1984. - Utilisation de géotextiles en stabilisation végétale des rives. Bulletin ARPEA. 123, : 51-63.

Lachat B. 1986. - Biotechnologie : Accélération de la croissance végétale avec des géotextiles / Ingenieurbiologie : Wachstumsbeschleunigung durch Anwendung von Geotextilien. Der Gartenbau. 25/86, : 1150-1152.

Lachat B. 1991a. - Le cours d'eau - Conservation, entretien et aménagement. Série aménagement et gestion $n^{\circ} 2$. Conseil de l'Europe. Strasbourg. 84 p.

Lachat B. 1991b. - Hydroécologie et génie biologique - les fondements de l'aménagement des cours d'eau. SIA Ingénieurs et architectes suisses. $24,:$ 503-510.

Lachat B. 1994. - Guide de protection des berges de cours d'eau en techniques végétales. Ministère de l'Environnement, Paris : 143 p.

Leuzinger Y. \& Lachat B. 1995. — Le génie biologique en question. Bull. Association pour le génie biologique. 3, : 3-7.

Lewis G. \& Williams G. 1984. - Rivers and wildlife Handbook: a guide to practices which further the conservation of wildlife on rivers. RSPB, RSNC : 296 p.
Maridet L. 1994. - La végétation rivulaire, facteur de contrôle du fonctionnement écologique des cours d'eau : influence sur les communautés benthiques et hyporhéiques et sur les peuplements de poissons dans trois cours d'eau du massif central. Thèse Université Claude Bernard, Lyon I : 294 p.

Morgan R.P.C. \& Rickson R. J. 1995. - Slope Stabilization and Erosion Control. A Bioengineering Approach. E \& FN Spon (Ed.) : $274 \mathrm{p}$.

Regione Emilia-Romagna 1993. - Manuale tecnico di ingegneria naturalistica : $256 \mathrm{p}$.

Schiechtl H. M. 1973. - Sicherungsarbeiten im Landschaftsbau. Grundlagen Lebende Baustoffe Methoden. Verlag Goerg. D. W. Callwey, München : $244 \mathrm{p}$.

Schlüter U. 1984. - Zur Geschichte der Ingenieurbiologie. Landschaft + Stadt.16(1/2): 2-9.

Thiéry E. 1891. - Restauration des montagnes, Correction des torrents. Reboisement. Baudry et Cie.( éd.) Paris.

Wasson J. G., Malavoi J.R., Maridet L., Souchion Y. \& Paulin L. 1995. - Impacts écologiques de la chenalisation des rivières. Cemagref, epteau, Ministère de l'environnement. Lyon et Paris. $168 \mathrm{p}$.

Zeh H. 1990. - Grenzen der Ingenieurbiologie. In Grundsätze und Beispiele der Ingenieurbiologie. Vortragstagung 7.12.90. Verein für Ingenieurbiologie, Zürich. : 33-37.

Zeh H., Roth H., Mosimann R., Schenker J., Lachat B. \& Dürler R. 1990. - Mesures de génie biologique dans l'aménagement des rives. Méthodes et exemples dans le canton de Berne. Direction des travaux publics du canton de Berne - Office des ponts et chaussées. $44 \mathrm{p}$. 